\title{
Variabilidad geográfica de mastografías de cáncer de mama en Jalisco, México, 2013-2015
}

\author{
Antonio Reyna-Sevilla* \\ Departamento de Salud Pública, División de Disciplinas para el Desarrollo, Promoción y Preservación de la Salud, Centro Universitario de Ciencias \\ de la Salud, Universidad de Guadalajara, Guadalajara, Jal., México
}

\begin{abstract}
Resumen
Un programa de detección organizado mediante mastografías podría contribuir a reducir la mortalidad por cáncer de mama (CaMa), por lo que es indispensable contar con evidencia para decidir hacia qué lugares dirigir dicha estrategia. Se realizó un diseño ecológico basado en estudios mastográficos del trienio 2013-2015, para examinar la variabilidad geográfica de resultados sospechosos y altamente sugestivos de malignidad en usuarias de un hospital oncológico de Guadalajara, Jalisco. Se estimaron tasas estandarizadas y riesgos relativos para cada categoría según municipio de residencia de las usuarias. De 1,150 mastografías, el 55.1\% reportó sospecha y el 44.9\% altamente sugestiva de malignidad. En Atoyac (zona sur) se registraron 78.6 casos sospechosos de malignidad por 100 mil mujeres $\geq 40$ años, mientras que en Ejutla (zona suroeste) fueron 150.7 casos altamente sugestivos de malignidad por 100 mil mujeres $\geq 40$ años. Sin embargo, el riesgo de detección en ambas categorías, hasta cuatro veces mayor, se extendió desde municipios de la zona suroeste hasta el noreste de Jalisco. La variabilidad geográfica de la detección sospechosa y altamente sugestiva de malignidad mostró los municipios donde se podrían dirigir programas de detección temprana de CaMa, según las usuarias atendidas en el hospital oncológico.
\end{abstract}

Palabras clave: Neoplasias de mama. Mamografía. Riesgo relativo. Epidemiología espacial. Sistemas de información geográfica.

\section{Geographical variability of mammograms for breast cancer in Jalisco, Mexico, 2013-2015}

\section{Abstract}

A organized screening program by mammograms could help reducing mortality from breast cancer, so evidence is essential to decide where to target this strategy. Ecological design based on mammographic studies from 2013-2015 period, to examine the geographic variability of suspicious and highly suggestive results of malignancy in users of an oncology hospital in Guadalajara, Jalisco. Standardized rates and relative risks for each category were estimated according to the users municipality of residence. Considering 1,150 mammograms, 55.1\% reported suspicious and $44.9 \%$ highly suggestive of malignancy. In Atoyac (southern area) 78.6 cases were reported with suspicious of malignancy per 100 thousand women $\geq 40$ years, while in Ejutla (southwestern area) were 150.7 highly suggestive cases of malignancy per 100 thousand women $\geq 40$ years. However, the detection risk in both categories, up to 4 times higher, spread from municipalities in the southwest to northeast

\section{Correspondencia:}

*Antonio Reyna-Sevilla

E-mail: gs.antonioreyna@gmail.com
Disponible en internet: 08-06-2021

Gac Mex Oncol. 2021;20(2):62-70

www.gamo-smeo.com (http://creativecommons.org/licenses/by-nc-nd/4.0/). 
of Jalisco. The geographical variability of detection with suspicious and highly suggestive of malignancy showed the municipalities where $B C$ early detection programs could be targeted, according to users treated at the oncology hospital.

Key words: Breast neoplasms. Mammography. Relative risk. Spatial epidemiology. Geographic Information System.

\section{Introducción}

En años recientes los tumores malignos se han colocado como una de las principales causas de mortalidad en población mexicana ${ }^{1}$. En el 2017, según el Instituto Nacional de Estadística y Geografía (INEGI)², estaban en tercer lugar tanto en hombres como mujeres; año en el cual se registraron 83,476 defunciones $^{3}$, por encima de enfermedades del hígado, accidentes de tránsito y homicidios, por mencionar algunas $^{2}$. Así, el cáncer de mama (CaMa) (C50) registró 6,854 decesos $^{2}$, esto es, un incremento del $8 \%$ $(n=550)$ en comparación con $2015^{4}$; principalmente en mujeres $>45$ años, y se colocó por encima de otros, como el cervicouterino ${ }^{2}$, lo cual ya había sido reportado desde el año $2006^{5}$. Más aún, de acuerdo con estimaciones de mortalidad por CaMa, para los próximos años se espera un continuo incremento en mujeres $>25$ años ${ }^{6}$.

Ante dicho panorama, se ha planteado que la falta de un programa de detección organizado ${ }^{6}$, capaz de realizar suficientes mastografías a la población blanco, proporcionar un diagnóstico y tratamiento adecuado, como lo establece la NOM-041-SSA2-2011 para la prevención, diagnóstico, tratamiento, control y vigilancia epidemiológica del $\mathrm{CaMa}^{7}$, podría explicar parcialmente la magnitud de la mortalidad por CaMa en México ${ }^{5}$, que ha sido documentada recientemente ${ }^{2-4}$. En EE.UU., por ejemplo, la disminución de la mortalidad a lo largo de varios años se ha asociado, en parte, a la implementación de estrategias de detección temprana mediante mastografía, junto con los avances en los tratamientos médicos ${ }^{8}$. Inclusive, algunos autores argumentan que la mortalidad también es consecuencia de los diagnósticos en fase avanzada, una problemática señalada en México desde $2003^{9}$; una de cada dos mujeres son clasificadas en estadios Ilb-IV6,10. Por ello se argumenta que un programa organizado de detección temprana de CaMa, como lo establece la NOM-041-SSA2-2011', junto con los suficientes recursos humanos, materiales y técnicos para asignar tratamientos, podría disminuir la mortalidad entre el $21^{6}$ y el $40 \%$; en comparación con otros métodos, como la autoexploración y el examen clínico, que hasta el momento no han demostrado el mismo impacto ${ }^{9}$.
Sin embargo, el Sistema Nacional de Salud no cuenta con los recursos suficientes para realizar tamizaje poblacional ${ }^{5} \mathrm{y}$, de ser necesario, atender clínicamente el CaMa en mujeres ${ }^{11}$, aunado a que la infraestructura disponible es subutilizada, lo cual ocasiona bajas coberturas en varios Estados del país ${ }^{5,12}$. Si bien es cierto que la identificación de factores de riesgo a nivel individual, como la edad o antecedentes familiares, permite definir grupos de población susceptibles de tamizaje ${ }^{7}$, también resulta importante determinar los lugares (vecindarios, municipios, regiones sanitarias) donde se deben focalizar los programas de detección temprana, lo cual implica no solo mejorar la distribución de los servicios de salud orientados a dicho propósito y aproximar geográficamente el tamizaje a los grupos de mayor riesgo (mujeres $\geq 40$ años) ${ }^{13}$, sino también incrementar sustancialmente la eficiencia de los programas ${ }^{13}$.

Por lo anteriormente expuesto, es indispensable optimizar los recursos disponibles relacionados con los programas de tamizaje, que en gran parte del país son limitados, y de esta manera intentar cumplir adecuadamente con la demanda de la detección temprana del CaMa en mujeres; particularmente en aquellos lugares asociados a un mayor riesgo de detección en categorías 4 o 5, según la clasificación Breast Imaging Report and Database System (BIRADS) ${ }^{6,14}$, dado que estas sugieren un resultado sospechoso o altamente sugestivo de malignidad de CaMa, respectivamente. Ante la falta de investigaciones en México, que involucren un enfoque epidemiológico y geográfico (epidemiología espacial $)^{15}$, se realizó un estudio en el Estado de Jalisco, cuyo objetivo fue examinar la variabilidad geográfica de mastografías sospechosas y altamente sugestivas de malignidad (BIRADS 4 y 5) 6,14 de CaMa, para identificar zonas de riesgo en las que se requiere focalizar o reforzar los programas de detección temprana de CaMa.

\section{Metodología}

Para lograr el objetivo planteado, se realizó un diseño ecológico en el que se incluyeron datos relacionados con el proceso de detección de CaMa en mujeres residentes del Estado de Jalisco, cuya atención médica se registró en un hospital especializado en el área 
oncológica, localizado en la ciudad de Guadalajara, durante el trienio 2013-2015; dicho hospital pertenece a la Secretaría de Salud de Jalisco, atiende a población abierta diagnosticada con diferentes tipos de cánceres y es uno de los principales centros de referencia de atención oncológica en el Occidente de México. Antes de iniciar la investigación se obtuvo la aprobación por el comité de ética e investigación del mismo hospital.

\section{Fuente y recolección de datos}

Los datos de interés se obtuvieron mediante un proceso de revisión de expedientes clínicos realizado en el hospital mencionado, garantizando la confidencialidad y anonimato de las usuarias. Se seleccionaron aquellos casos cuyo estudio de mastografía reportó la categoría sospechoso y altamente sugestivo de malignidad, esto es, BIRADS 4 y 5 , respectivamente, de usuarias $\geq 40$ años atendidas en el periodo de estudio y residentes del Estado de Jalisco ${ }^{6,14}$. En la fase de recolección de datos se realizó una estandarización y evaluación de la confiabilidad, lo cual se explica en detalle en otro manuscrito ${ }^{16}$. Brevemente, este proceso se llevó a cabo para verificar que la información capturada y analizada correspondió con lo reportado en los expedientes clínicos incluidos en el estudio, y de esta manera minimizar posibles sesgos en el diseño.

\section{Georreferenciación de datos y unidad espacial}

Una vez terminada la etapa de recolección, los datos se integraron en el Marco Geoestadístico Municipal de Jalisco mediante el Sistema de Información Geográfica (SIG) QGIS (versión 3.10), lo cual se refiere a la delimitación política administrativa de 125 municipios según el Instituto Nacional de Estadística y Geografía $(\text { INEGI })^{17}$. Esta etapa fue útil para vincular los datos recolectados con un referente espacial, agrupar los municipios según las 13 regiones sanitarias en el Estado, así como determinar la frecuencia de ambas categorías BIRADS por municipio (datos agregados), para posteriormente estimar tasas estandarizadas por edad, municipio y año.

\section{Cálculo de indicadores y análisis geoestadístico}

Con base en la delimitación municipal de Jalisco, se estimaron tres indicadores espaciales para el trienio de estudio: 1) casos sospechosos y 2) altamente sugestivos de malignidad por 100 mil mujeres $\geq 40$ años, así como 3) riesgo relativo (exceso de riesgo) para cada categoría. La estimación de las tasas se obtuvo mediante método directo ${ }^{18}$, al dividir el total de usuarias $\geq 40$ años cuyo estudio de mastografía reportó BIRADS 405 por municipio (2013-2015), entre la población femenina $\geq$ 40 años reportada a nivel municipal durante el mismo periodo, según datos oficiales de la Dirección General de Información en Salud (DGIS) ${ }^{19}$. Ambos indicadores se agruparon en cuartiles para identificar los valores superiores e inferiores atípicos según municipio. El riesgo relativo, por otro lado, consistió en comparar, mediante una razón, cada tasa estimada por unidad espacial entre el promedio municipal obtenido en el trienio; los tres indicadores fueron calculados mediante el software SIG GeoDa (versión 1.14).

A partir de lo anterior, se realizó un análisis geoestadístico ${ }^{20}$ para examinar no solo la magnitud, sino también la variabilidad geográfica de los indicadores, y de esta manera determinar zonas de mayor o menor riesgo. Adicionalmente, se realizaron mediciones de tendencia espacial ${ }^{20,21}$ para identificar los municipios donde se concentró el mayor riesgo de detección de casos sospechosos o altamente sugestivos de malignidad. Los resultados se presentaron mediante mapas elaborados en los SIG antes mencionados.

\section{Resultados}

Durante el trienio de estudio se revisaron 1,150 (N) expedientes clínicos relacionados con usuarias diagnosticadas con CaMa; de estas, el 55.1\% ( $n=633$ ) fueron casos sospechosos de malignidad (BIRADS 4), cuya edad promedio fue 52.4 años (desviación estándar $[D E] \pm 11.9$ ); en tanto que la categoría altamente sugestiva de malignidad (BIRADS 5) fue reportada en el $44.9 \%(n=517)$ de los casos, sin embargo, la edad promedio de este grupo fue diferente $(p<0.05)$ al anterior, esto es, 55.4 años (DE \pm 11.9).

En la figura 1 se observa la distribución de las usuarias clasificadas en BIRADS 4 durante el trienio 20132015 según municipio de residencia. A partir de ello, se identificó que el total de estas residían solo en el $68.8 \%(n=86)$ de los municipios de Jalisco, cuya frecuencia de casos osciló entre 1 (27 municipios) y 164 (Guadalajara). Además, los municipios localizados en la zona centro, pertenecientes a las regiones sanitarias Zapopan, Tonalá, Tlaquepaque y Guadalajara, fueron los que concentraron una mayor proporción de casos, esto es, hasta 6 de cada $10(n=389)$. 


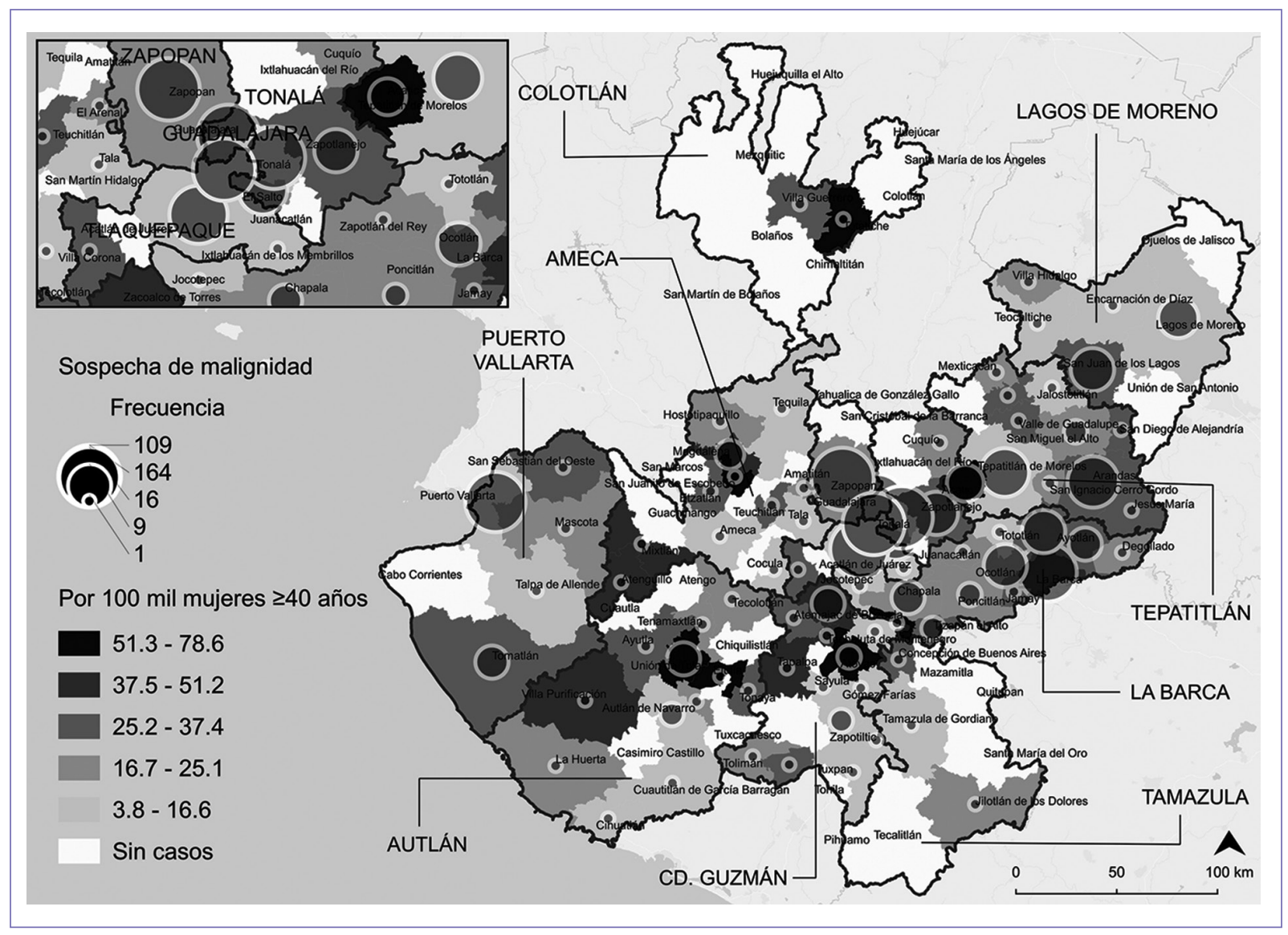

Figura 1. Frecuencia de casos $(n=633$ ) sospechosos de malignidad según municipio de residencia en Jalisco, 20132015 (elaboración propia a partir de trabajo de campo y el Marco Geoestadístico Nacional, 2018 17).

Respecto a los valores estandarizados a nivel municipal, solo dos municipios registraron un valor superior atípico: Ejutla (zona suroeste) con 75.3 y Atoyac (zona sur) con 78.6 casos con sospecha de malignidad por 100 mil mujeres $\geq 40$ años, en comparación con los valores más bajos registrados en la región sanitaria Lagos de Moreno (Fig. 1). Sin embargo, el mayor riesgo de detección de BIRADS 4 se extendió hacia aquellos municipios localizados de suroeste a noreste de Jalisco, lo cual está representado por la elipse de la figura 2; como resultado, Acatic, Atoyac, Ejutla, Totatiche y Unión de Tula (en color negro) mostraron un riesgo relativo hasta cuatro veces mayor que el promedio municipal (Poncitlán: 18.9 casos sospechosos de malignidad por 100 mil mujeres $\geq 40$ años).

Por otra parte, considerando el área de residencia de las usuarias clasificadas como altamente sugestivo de malignidad (Fig. 3), se identificó que el total residía en el $65.6 \%(n=82)$ de los municipios de Jalisco, con una frecuencia de casos entre 1 (28 municipios) y 130
(Guadalajara); pero en comparación con los demás, los municipios del centro (regiones sanitarias Zapopan, Tonalá, Tlaquepaque y Guadalajara ) y noreste (región sanitaria Tepatitlán) concentraron hasta el 62\% ( $n=320)$ de las usuarias clasificadas en dicha categoría.

En la figura 3 se observa la magnitud y variabilidad de la detección altamente sugestiva de malignidad, cuyos valores superiores atípicos fueron: 71.8 (Santa María del Oro, región sanitaria Tamazula), 73.3 (Cañadas de Obregón, región sanitaria Tepatitlán), 87.5 (Valle de Guadalupe, región sanitaria Tepatitlán) y 150.7 (Ejutla, región sanitaria Autlán) casos por 100 mil mujeres $\geq 40$ años; a diferencia de los valores más bajos registrados en las regiones sanitarias Puerto Vallarta y Autlán. No obstante, el riesgo relativo fue hasta cuatro veces mayor en municipios del suroeste y noreste del Estado (elipse) (Fig. 4), esto es, Ejutla, Santa María del Oro, Valle de Guadalupe y Cañadas de Obregón; en comparación con el promedio municipal de 15.8 


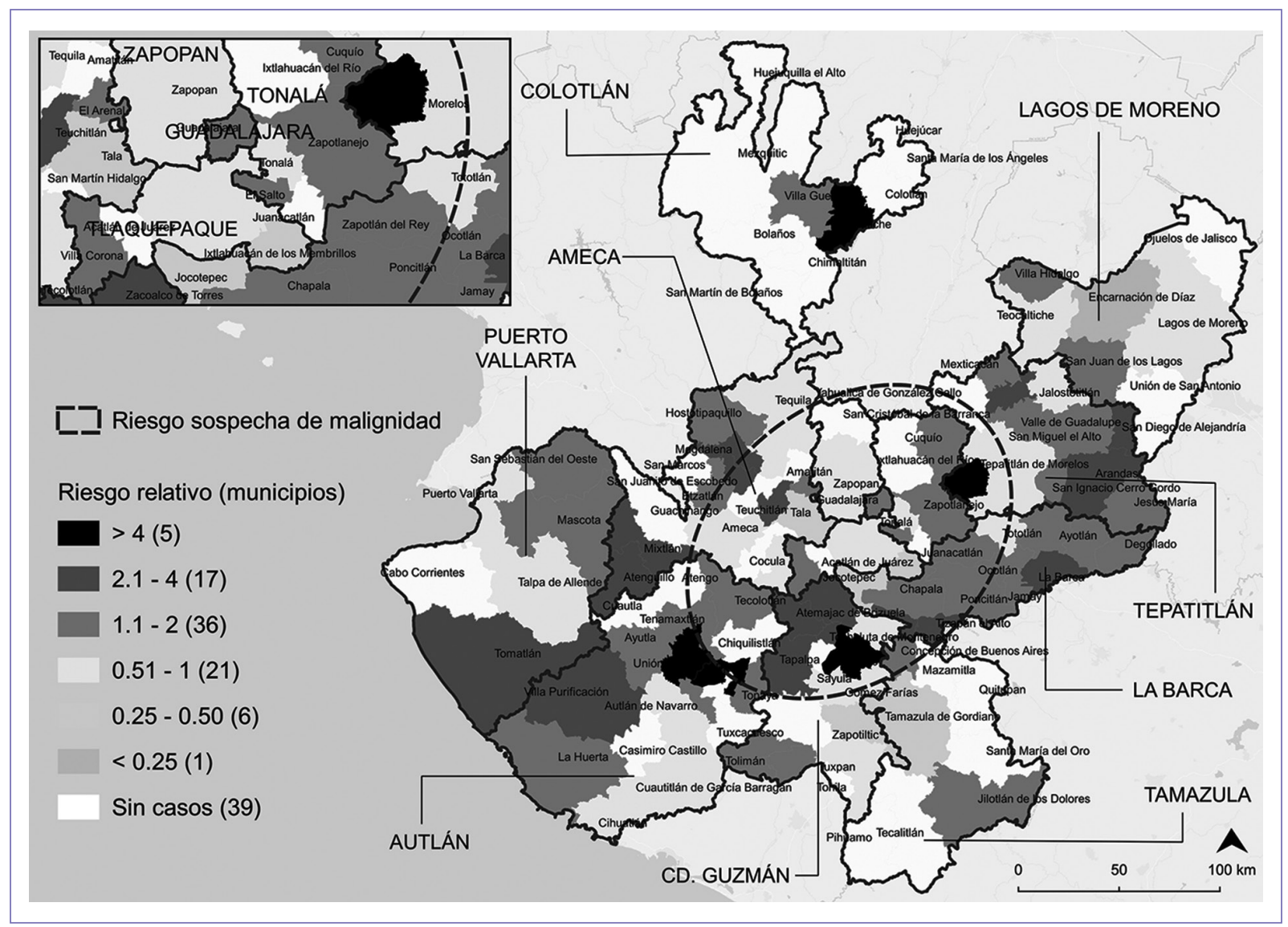

Figura 2. Riesgo relativo de detección de casos sospechosos de malignidad según municipio de residencia de las usuarias en Jalisco, 2013-2015 (elaboración propia a partir de trabajo de campo y el Marco Geoestadístico Nacional, $2018^{17}$ ).

casos altamente sugestivos de malignidad por 100 mil mujeres $\geq 40$ años registrado en Encarnación de Díaz.

\section{Discusión}

La variabilidad geográfica de la detección de CaMa mediante mastografía mostró municipios con un riesgo hasta cuatro veces mayor en comparación con otras áreas del Estado de Jalisco, por lo que el área administrativa y médica del hospital especializado en oncología, como parte del Sistema Nacional de Salud, podría contribuir a reforzar programas de detección temprana en los municipios de residencia y de mayor riesgo según las usuarias que generalmente son atendidas. Aunado a que las categorías analizadas BIRADS 4 y 5 indican que la mastografía parece ser una opción de la evaluación diagnóstica y no un método de detección temprana de $\mathrm{CaMa}$, como lo establece la NOM-041-SSA2-2011?.
Sin duda, uno de los principales propósitos de los programas de tamizaje es mejorar los procesos de detección, diagnóstico de CaMa y favorecer el uso de terapias efectivas en las mujeres ${ }^{6,7,13}$. Sin embargo, se han documentado diversas problemáticas importantes que impiden lograr tal propósito a nivel nacional. Por un lado, se ha reportado una cobertura de mastografías limitada ${ }^{5}$, que oscila entre el 11.7 y el $22.8 \%$ para los grupos de mujeres 40-49 y 50-69 años, respectivamente; según el área de residencia, también se han encontrado diferencias entre el 19.1\% (40-49 años) en zona rural y el 17.7\% (50-69 años) en zona urbana ${ }^{12}$. $\mathrm{Si}$ a esto agregamos que el diagnóstico de CaMa en México se realiza generalmente en fases avanzadas $^{6,10}$, resulta importante el análisis de las categorías BIRADS reportadas por estudios mastográficos al igual que la edad en la que esto ocurre. De acuerdo con los resultados aquí presentados, hubo diferencias significativas $(p<0.05)$ entre las categorías BIRADS 4 y 5 


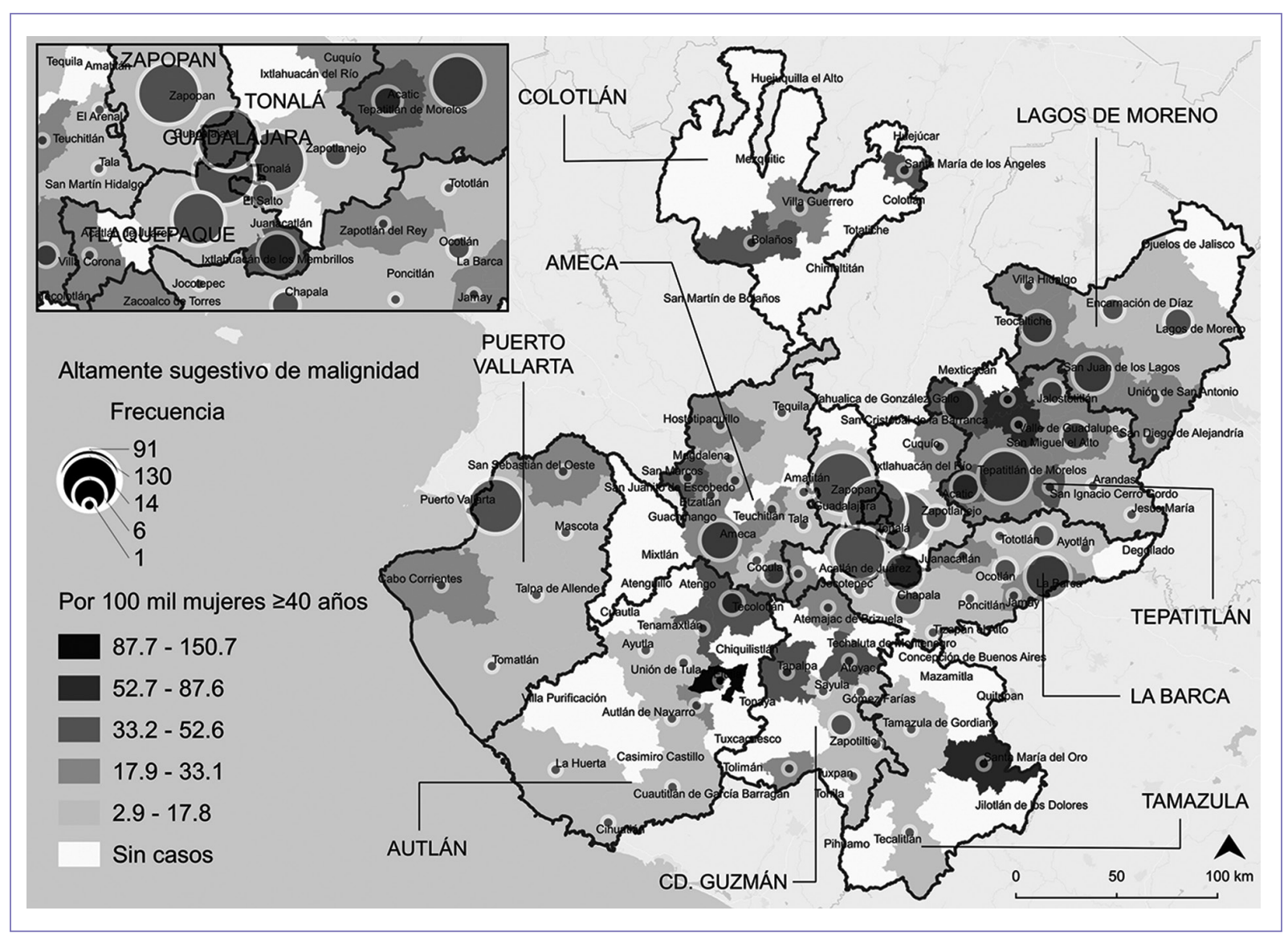

Figura 3. Frecuencia de casos $(\mathrm{n}=517$ ) altamente sugestivos de malignidad según municipio de residencia en Jalisco, 2013-2015 (elaboración propia a partir de trabajo de campo y el Marco Geoestadístico Nacional, $2018^{17}$ ).

según el promedio de edad, esto es, 52 y 55 años, respectivamente; un rango (de riesgo ${ }^{6}$ similar a lo reportado en otro estudio ${ }^{10}$. Por ello, es posible que los lineamientos establecidos en la NOM-041-SSA2-20117, respecto al proceso de detección en mujeres según el grupo de edad al que pertenecen, por ejemplo autoexploración, examen clínico o programas de tamizaje, no estén funcionando en algunos municipios localizados en la zona suroeste y noreste de Jalisco, que podrían contribuir a incrementar el número de estudios mastográficos como método de detección temprana.

La importancia de un tamizaje poblacional efectivo radica, entre otros aspectos, en incrementar la detección en etapas preclínicas así como la referencia oportuna y, eventualmente, reducir las tasas de mortalidad por CaMa en mujeres ${ }^{6,8,9}$; caso contrario, se incrementa el costo de la atención médica y disminuyen considerablemente las posibilidades de supervivencia ${ }^{6,9}$. Sin embargo, en Jalisco se ha reportado una cobertura de mastografías $<30 \%{ }^{12}$, es decir, dos veces por debajo de la recomendación de la Organización Mundial de la Salud de tamizar, por lo menos, al 70\% de la población en riesgo'22; lo cual podría ser explicado en parte por las carencias de infraestructura en el Sistema Nacional de Salud para realizar las suficientes mastografías en mujeres $\geq 40$ años ${ }^{5,7}$. Ante esta situación, se hace necesario mejorar o reforzar un programa de detección organizado como establece la NOM-041-SSA2-20117, como parte de las medidas de prevención secundaria $^{7,23}$. Por consiguiente, la identificación de grupos y áreas de mayor riesgo adquiere importancia para determinar en quiénes y en dónde se requiere focalizar, por ejemplo, los programas de detección organizados en función del perfil de usuarias que generalmente son atendidas en hospitales que brindan atención oncológica.

En este sentido, el enfoque de la epidemiología espacial, mediante el mapeo y análisis de datos asociados a la detección y diagnóstico de $\mathrm{CaMa}^{15}$, contribuye en la identificación de áreas de riesgo que podrían ser 


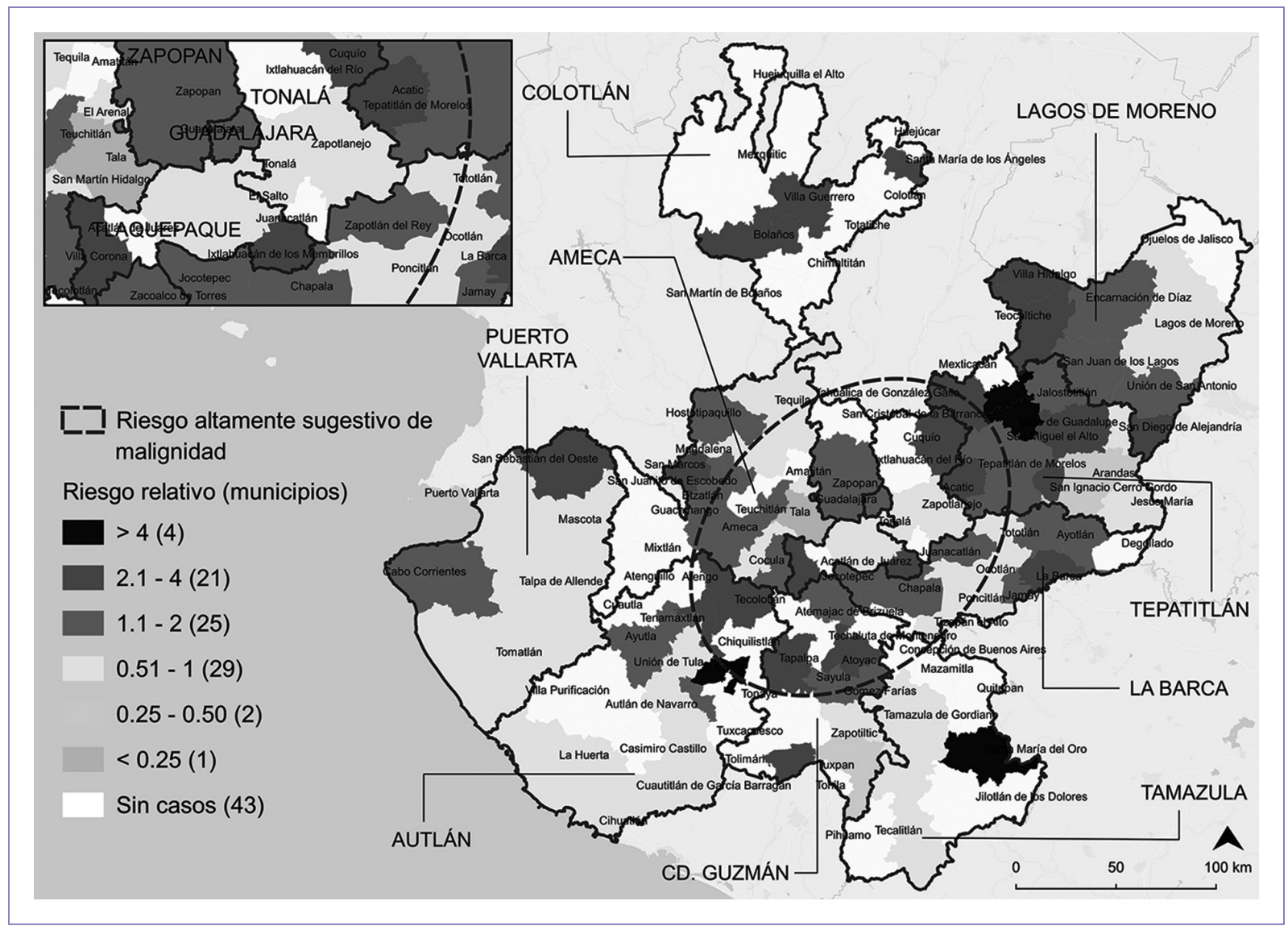

Figura 4. Riesgo relativo de detección de casos altamente sugestivos de malignidad según municipio de residencia de las usuarias en Jalisco, 2013-2015 (elaboración propia a partir de trabajo de campo y el Marco Geoestadístico Nacional, $2018^{17}$ ).

beneficiadas mediante intervenciones focalizadas; ya sea que estén relacionadas con fases avanzadas de la enfermedad, o bien tasas de mortalidad superiores a lo esperado, como se ha realizado en otros países ${ }^{24,25}$. Más aún, la identificación de áreas de riesgo podría adecuarse a diversas escalas territoriales, por ejemplo, unidades censales, vecindarios, municipios, regiones sanitarias; en las cuales, incluso, se dispone de información sociodemográfica o de servicios de salud que podría complementar un análisis con enfoque epidemiológico espacial.

En efecto, una de las enfermedades no transmisibles respecto a la cual se ha desarrollado mayor investigación desde el enfoque epidemiológico espacial ha sido el cáncer ${ }^{15,24}$. El potencial de dicho enfoque radica, entre otros aspectos, en examinar variaciones y patrones espaciales, mediante el uso de tecnología geoespacial, que podrían apoyar en el control y vigilancia epidemiológica del CaMa, identificar lugares donde se necesita desarrollar más investigación (epidemiológica) o focalizar intervenciones ${ }^{25}$, así como mejorar la efectividad del tamizaje poblacional, por ejemplo, mediante la aproximación geográfica de este tipo de servicios de salud con la finalidad de incrementar la detección precoz de casos, aumentar la supervivencia, mejorar la calidad de vida y, eventualmente, reducir las tasas de mortalidad por $\mathrm{CaMa}^{9}$.

Considerando el diseño epidemiológico presentado, es necesario señalar algunas limitaciones. Por un lado, el uso de una fuente secundaria limitó la posibilidad de conocer si el estudio mastográfico de las usuarias fue utilizado como método de tamizaje o diagnóstico, según los criterios de la NOM-041-SSA2-20117; dado que esto podría aportar evidencia respecto al proceso de referencia, diagnóstico y tratamiento de las usuarias atendidas en el hospital de atención oncológica. Inclusive, al ser un centro de referencia estatal, que también recibe pacientes de otras entidades federativas, es 
probable que la mayoría de los estudios mastográficos se relacionen con categorías BIRADS sospechosas y altamente sugestivas de malignidad en comparación con las demás.

Por otro lado, no fue posible determinar las subcategorías establecidas en la clasificación 4 según $\mathrm{BI}$ RADS $^{6,14}$, lo que ciertamente hubiera aportado resultados más específicos sobre lo reportado en los estudios mastográficos. Sin embargo, ello depende en gran parte de la accesibilidad y disponibilidad de los datos, lo que, por cierto, explica que este diseño no fue de base poblacional, esto es, no se incluyeron usuarias del sector privado u otros servicios de salud (Instituto Mexicano del Seguro Social, Instituto de Seguridad y Servicios Sociales de los Trabajadores del Estado, entre otros). Aun así, un aspecto que destacar en el diseño presentado es el análisis geográfico respecto a datos clínicos que generalmente no son accesibles, o bien son utilizados únicamente en el ámbito clínico. Por lo tanto, los resultados aportaron otra perspectiva a la problemática que actualmente representa el proceso de detección del CaMa en mujeres en el Estado de Jalisco, que bien podría complementar las actividades en el ámbito administrativo y médico en los hospitales que proporcionan atención oncológica.

\section{Conclusiones}

En conclusión, los resultados de estudios mastográficos de usuarias atendidas en un hospital oncológico (2013-2015) permitieron identificar, por un lado, diferencias en la edad promedio de detección con sospecha y altamente sugestiva de malignidad de CaMa, esto es, entre 52 y 55 años, respectivamente. Por otro lado, se identificaron los municipios cuyo riesgo de detección en ambas categorías se incrementó hasta cuatro veces, esto es, zona suroeste y noreste de Jalisco. Por ello, la evidencia podría contribuir a determinar cuáles son los municipios donde se necesitan focalizar programas de tamizaje poblacional, como una alternativa para mejorar los procesos de detección y, por consiguiente, incrementar la oportunidad de un diagnóstico temprano de CaMa en las mujeres que son atendidas en el hospital oncológico que forma parte del Sistema Nacional de Salud.

\section{Financiamiento}

Este manuscrito fue generado como parte de la investigación de una tesis doctoral, cuyo apoyo económico correspondió a una beca otorgada por el Consejo
Nacional de Ciencia y Tecnología (CONACYT) en relación con el Programa Nacional de Posgrados de Calidad (PNPC).

\section{Conflicto de intereses}

Los autores declaran no tener conflicto de intereses alguno.

\section{Responsabilidades éticas}

Protección de personas y animales. Los autores declaran que para esta investigación no se han realizado experimentos en seres humanos ni en animales.

Confidencialidad de los datos. Los autores declaran que en este artículo no aparecen datos de pacientes.

Derecho a la privacidad y consentimiento informado. Los autores declaran que en este artículo no aparecen datos de pacientes.

\section{Bibliografía}

1. DeSantis CE, Bray F, Ferlay J, Lortet-Tieulent J, Anderson BO, Jemal A. International variation in female breast cancer incidence and mortality rates. Cancer Epidemiol Biomarkers Prev. 2015;24(10):1495-506.

2. Instituto Nacional de Estadística y Geografía (INEGI). Características de las defunciones registradas en México durante 2017 [Internet]. Ciudad de México; 2018 [consultado: 8 de mayo de 2020]. Disponible en: https:// www.inegi.org.mx/contenidos/saladeprensa/boletines/2018/EstSociodemo/DEFUNCIONES2017.pdf

3. International Agency for Research on Cancer (IARC). Mexico Source: Globocan 2018, Vol. 283, The Global Cancer Observatory [Internet]. Lyon; 2019 [consultado: 14 de julio de 2020]. Disponible en: https://gco. iarc.fr/today/data/factsheets/populations/484-mexico-fact-sheets.pdf

4. Aldaco-Sarvide F, Pérez-Pérez P, Cervantes-Sánchez G, Torrecillas-Torres $L$, Erazo-Valle-Solís AA, Cabrera-Galeana $P$, et al. Mortality from cancer in Mexico: 2015 update. Gac Mex Oncol. 2018;17(1):28-34.

5. Uscanga S, Torres G, Ángeles A, Domínguez R, Lazcano E. Indicadores del proceso de tamizaje de cáncer de mama en México: un estudio de caso. Salud Publica Mex. 2014;56(5):528-37.

6. Cárdenas-Sánchez J, Bargalló-Rocha E, Erazo Valle A, Poitevin Chacón A, Valero Castillo V, Pérez Sánchez V. Consenso Mexicano sobre diagnóstico y tratamiento del cáncer mamario. Octava revisión. Colima 2019. Gac Mex Oncol. 2019;18:141-231.

7. Secretaría de Salud. Norma Oficial Mexicana NOM-041-SSA2-2011 para la prevención, diagnóstico, tratamiento, control y vigilancia epidemiológica del cáncer de mama [Internet]. México: Diario Oficial de la Federación; 2011 [citado el 13 de abril de 2020]. Disponible en: http://dof.gob.mx/ nota_detalle.php?codigo $=5194157$ \&fecha $=09 / 06 / 2011$

8. Berry DA, Cronin KA, Plevritis SK, Fryback DG, Clarke L, Zelen M, et al. Effect of screening and adjuvant therapy on mortality from breast cancer. N Engl J Med. 2005;353(17):1784-92.

9. Montemayor A. Tamizaje por mamografía en el cáncer de mama. En: Lazcano E, Escudero P, Uscanga S, editores. Cáncer de mama Diagnóstico, tratamiento, prevención y control. Instituto. Cuernavaca: SPM Ediciones Ciencia; 2014. pp. 285-90.

10. Maffuz A, Labastida S, Espejo A, Rodríguez S. Características clinicopatológicas del cáncer de mama en una población de mujeres en México. Cir Cir. 2017;85(3):201-7

11. Bargalló E, Ruvalcaba-Limón E, Quintero-Beuló G, Reséndiz-Colosia JA. Recursos para la atención de pacientes con cáncer de mama en México. Gac Mex Oncol. 2019;18(3):121-6.

12. Torres-Mejía G, Ortega-Olvera C, Ángeles-Llerenas A, Villalobos-Hernández AL, Salmerón-Castro J, Lazcano-Ponce $E$, et al. Patrones de utilización de programas de prevención y diagnóstico temprano de cáncer en la mujer. Salud Publica Mex. 2013;55(2):241-8. 
13. Andrews HF, Kerner JF, Zauber AG, Mandelblatt J, Pittman J, Struening E. Using census and mortality data to target small areas for breast, colorectal and cervical cancer screening. Am J Public Health. 1994;84(1):56-61.

14. Camacho C, Espíndola V. Actualización de la nomenclatura BI-RADS ${ }$ por mastografía y ultrasonido. An Radiol México. 2018;17:100-8.

15. Roquette R, Painho M, Nunes B. Spatial epidemiology of cancer: a review of data sources, methods and risk factors. Geospat Health. 2017:12(1):23-35.

16. Reyna A, Ramos IM, González ME, Morales A, Valdez RM, Mora D. Evaluación de la confiabilidad de datos recolectados a partir de expedientes clínicos de cáncer de mama. UniverSalud. 2019;15(29):23-30.

17. Instituto Nacional de Estadística y Geografía. Marco Geoestadístico Nacional, junio 2018 [Internet]. México: Instituto Nacional de Estadística y Geografía [consultado: 25 de abril de 2019]. Disponible en: https://www. inegi.org.mx/app/biblioteca/ficha.html?upc $=889463592587$

18. Hernández M, Lazcano E, Oropeza C, editores. Epidemiología. Diseño y análisis de estudios. 2. ${ }^{\text {a }}$ ed. Cuernavaca: Instituto Nacional de Salud Pública; 2015.

19. Cubos dinámicos-Población (Proyecciones). Proyecciones CONAPO versión Censo 2010 [Internet]. México: Gobierno de México, Dirección General de Información en Salud (DGIS) [consultado: 13 de agosto de 2018]. Disponible en: http://www.dgis.salud.gob.mx/contenidos/basesdedatos/bdc_poblacion_gobmx.html
20. Shekhar S, Li Y, Ali RY, Eftelioglu E, Tang X, Jiang Z. Spatial and spatiotemporal data mining. En: Huang B, editor. Comprehensive Geographic Information Systems. Elsevier; 2018. pp. 264-86.

21. Wong D, Wang F. Spatial Analysis Methods. En: Huang B, editor. Comprehensive Geographic Information Systems. 1. a ed. Elsevier; 2017.

22. World Health Organization. Cancer control: knowledge into action: WHO guide for effective programmes: Early detection [Internet]. World Health Organization; 2007 [consultado: 12 de mayo de 2020]. Disponible en: http://whqlibdoc.who.int/publications/2007/9241547338_eng.pdf?ua $=1$

23. Stewart BW, Wild C. World cancer report 2014 [Internet]. Lyon: International Agency for Research on Cancer, World Health Organization; 2014 [citado: 20 de septiembre de 2018]. Disponible en: http://publications.iarc. fr/Non-Series-Publications/World-Cancer-Reports/World-Cancer-Report-2014

24. Kerner JF, Andrews H, Zauber A, Struening E. Geographically-based cancer control: Methods for targeting and evaluating the impact of screening interventions on defined populations. J Clin Epidemiol. 1988;41(6):543-53

25. Tatalovich Z, Zhu L, Rolin A, Lewis DR, Harlan LC, Winn DM. Geographic disparities in late stage breast cancer incidence: Results from eight states in the United States. Int J Health Geogr. 2015;14(1):31. 